References:

[1] Benatti FB, Pedersen BK. Exercise as an anti-inflammatory therapy for rheumatic diseases-myokine regulation. Nat Rev Rheumatol 2015;11:86-97.

[2] Hagströmer M, Oja P, Sjöström M. The International Physical Activity Questionnaire (IPAQ): a study of concurrent and construct validity. Public Health Nutr 2006;9:755-62.

[3] Young NA, Jablonski K, Sharma J, Thomas E, Snoad B, Hampton J, et al. Low and Moderate Intensity Exercise Suppresses Inflammatory Responses in an Acute Mouse Model of Gout and Suggests Therapeutic Efficacy [abstract]. Arthritis Rheumatol. 2017;69 (suppl 10).

Disclosure of Interests: Naomi Schlesinger Grant/research support from: Pfizer, AMGEN, Consultant of: Novartis, Horizon Pharma, Selecta Biosciences, Olatec, IFM Therapeutics, Mallinckrodt Pharmaceuticals, Speakers bureau: Takeda, Horizon, Kyle jablonski: None declared, Emmy schwarz: None declared, Nicholas Young: None declared

DOI: 10.1136/annrheumdis-2020-eular.490

\section{AB0934 DUAL-ENERGY COMPUTED TOMOGRAPHY IN GOUT PATIENTS: IS IT USEFUL IN GENERAL PRACTICE?}

Y. Sherman ${ }^{1}$, P. Lipsky ${ }^{2}$, M. Bramwit ${ }^{1}$, N. Schlesinger ${ }^{1} .{ }^{1}$ Rutgers Robert Wood Johnson Medical School, New Brunswick, United States of America; ${ }^{2}$ RILITE Research Institute, Charlottesville, VA, United States of America

Background: Dual-Energy CT (DECT) has high sensitivity and specificity for detecting monosodium urate (MSU) crystal deposition. Although widely used in research, few studies have evaluated the usefulness of DECT in clinical practice. Objectives: To evaluate the use of DECT in a clinical setting and determine its utility

Methods: We retrospectively evaluated the records of all patients referred for DECT scans over a 6.5-year period. Patient charts were reviewed for clinical features.

Results: 113 patients (17.5/yr) received DECT evaluation at a university hospital over the study period (234 scans). All were referred by rheumatologists. Medical records were available for 69 patients (134 scans), including 44 males and 25 females (mean age 62 (SD, 12.9, range: 34-85 yrs). Mean duration of gout was 6.7 (SD, 8.1) yrs. DECT was ordered to evaluate known gout $(36 / 69,52.1 \%)$, suspected gout $(32 / 69,46.4 \%)$, and suspected calcium pyrophosphate (CPP) disease (1/69, 1.4\%). 32/69 (46.4\%) of patients were on urate-lowering therapy. $61 \%$ (42/69) had MSU crystal and none had CPP deposition. Mean MSU volume was $1.6 \mathrm{cc}$ (SD, $5.2 \mathrm{cc}$; range: $0.01-35 \mathrm{cc}$.) The joints imaged were feet/ankles $(80 / 134,60 \%)$ and hands/wrists $(53 / 134,40 \%) .23 / 33(69.7 \%)$ patients with DECT positivity had elevated serum urate (SU) levels $>6 \mathrm{mg} / \mathrm{dL}$; however, elevated SU was not significantly associated with DECT positivity (odds ratio (OR) $1.9,95 \% \mathrm{Cl}: 0.59-5.95, \mathrm{p}=0.28$ )

For patients with positive scans, mean gout duration from first known flare was 9.5 (SD, 8.8) yrs.

Among patients who had scans completed within $1 \mathrm{yr}$ of the first known gout flare, $1 / 10$ were positive (10\%); $4 / 16$ within $2 \mathrm{yrs}(25 \%)$; and $8 / 21$ within $3 \mathrm{yrs}$ $(29.6 \%)$.

Of patients with positive DECT scans, $24 / 42(57 \%)$ had symmetric distribution of MSU crystal deposition: 10/24 (42\%) hands and 14/24 (58\%) feet; with gout duration of 7.9 (SD, 8.0) yrs.

Conclusion: DECT was infrequently utilized and only by rheumatologists. Only $60 \%$ of patients referred for DECT scanning because of known or suspected gout had MSU deposition. DECT was uncommonly positive in patients with a 1-3 yr history of gout. When positive, the MSU crystal deposition was symmetrical in most gout patients. DECT scans, while important in furthering our understanding of gout biology, are not routinely used in general practice and often do not provide important decision support information. Establishment of practice guidelines might be important in developing more appropriate utilization of DECT.

Disclosure of Interests: Yekaterina Sherman: None declared, Peter Lipsky Consultant of: Horizon Therapeutics, Mark Bramwit: None declared, Naomi Schlesinger Grant/research support from: Pfizer, AMGEN, Consultant of: Novartis, Horizon Pharma, Selecta Biosciences, Olatec, IFM Therapeutics, Mallinckrodt Pharmaceuticals, Speakers bureau: Takeda, Horizon

DOI: 10.1136/annrheumdis-2020-eular.3833

\section{AB0935 \\ HYDROLYSIS OF INORGANIC PYROPHOSPHATE IN HUMAN SYNOVIAL FLUID BY IMMOBILIZED PYROPHOSPHATASES}

A. Valueva ${ }^{1}$, R. Romanov ${ }^{2}$, S. Mariasina ${ }^{2}$, M. Eliseev ${ }^{3}$, E. Rodina ${ }^{2} .{ }^{1}$ Pavlov Ryazan State Medical University, Ryazan, Russian Federation; ${ }^{2}$ Lomonosov Moscow State University, Moscow, Russian Federation; ${ }^{3}$ V.A.Nasonova Research Institute of Reumatology, Moscow, Russian Federation
Background: Calcium pyrophosphate deposition (CPPD) disease, alternatively known as pseudogout, is a type of arthropathy caused by a deposition of microcrystals of calcium pyrophosphate (CaPPi) in joint tissues, particularly in fibrocartilage and hyaline cartilage. Up to date, specific treatment of CPPD disease has not been developed. Conventional therapy is focused on anti-inflammatory and immunosuppressive treatment aimed at the prevention of acute symptoms. Previously we suggested for this purpose inorganic pyrophosphatases (PPases) immobilized on nanodiamonds of detonation synthesis (NDs) [1]. We synthesized and characterized conjugates of NDs with PPases from Escherichia coli and Mycobacterium tuberculosis (Ec-PPase or Mt-PPase) using amino-substituted NDs with (ND-L) or without linker (ND-NH N $_{2}$. The conjugates showed high enzymatic activity in the in vitro model system simulating the ionic composition of synovial fluid

Objectives: The present work is focused on testing enzyme-based agents for the partial dissolution of calcium pyrophosphate deposits in the synovial fluid of patients with CPPD disease.

Methods: PPase activity of soluble and immobilized PPases was evaluated as the rate of PPi decrease with time. $1 \mathrm{mM} \mathrm{PP}$, was added to the synovial fluid of patients with CPPD containing the samples of PPases, and the amount of PP in the samples was determined every $2-4$ hours using ${ }^{31} \mathrm{P}$ NMR spectroscopy. Results: We demonstrated the possibility of differential detection of pyrophosphate $\left(\mathrm{PP}_{\mathrm{j}}\right)$ among the spectrum of phosphorus-containing compounds in the synovial fluid and used the rate of decrease of exogenous PP. to estimate hydroIytic activity of PPases and PPase-based conjugates. The hydrolysis followed the first order reaction kinetics and the rate constants by different samples of PPases are presented in Table 1. Non-enzymatic hydrolysis of exogenous PP was quite moderate or not observed at all. Ec-PPase (both soluble and immobilized) showed very low hydrolytic activity with the residual PP, levels above $80 \%$. However, all samples of Mt-PPase showed significant hydrolytic activity. In two cases of three, PP was almost completely hydrolyzed within 20 hours. The maximum activity $\left(2.24{\left.\mathrm{U} \cdot \mathrm{mg}^{-1}\right)}^{-1}\right.$ was shown by Mt-PPase immobilized on ND-L. Hydrolytic activity for all studied enzymes is only $1-2 \%$ of such values under optimal in vitro conditions, most probably due to the inhibiting effect of calcium in synovial fluid. Nevertheless, the activity of Mt-PPase-based samples was comparable with the values typical for some classes of hydrolases. It allows us to consider the suggested PPase-based materials as promising agents for the hydrolysis of $\mathrm{PP}_{\mathrm{i}}$ in the joint tissues in vivo.

Table 1. PP, hydrolysis in the synovial fluid by soluble or immobilized PPases.

\begin{tabular}{lccc}
\hline Samples & & $k, \mathrm{hr}^{-1}$ & $A, \mathrm{U} \cdot \mathrm{mg}^{-1}$ \\
\hline Control & - & $0.010 \pm 0.001$ & 0 \\
\hline & $\mathrm{ND}^{-N}{ }_{2}$ & 0 & 0 \\
Ec-PPase & ND-L & $0.013 \pm 0.002$ & 0 \\
& Soluble & $0.025 \pm 0.003$ & $0.33 \pm 0.04$ \\
Mt-PPase & Immobilized on ND-NH $_{2}$ & 0 & 0 \\
& Immobilized on ND-L $^{2}$ & $0.017 \pm 0.003$ & $0.22 \pm 0.04$ \\
& Soluble & $0.10 \pm 0.01$ & $1.3 \pm 0.1$ \\
& Immobilized on ND-NH & $0.04 \pm 0.01$ & $0.53 \pm 0.05$ \\
& Immobilized on ND-L & $0.17 \pm 0.02$ & $2.2 \pm 0.3$ \\
\hline
\end{tabular}

Conclusion: In this work, we applied the ${ }^{31} \mathrm{P}$ NMR spectroscopy to estimate the quantity of $P$ and $P P$ in synovial fluids of patients with CPPD disease. The conjugates of bacterial PPases with detonation nanodiamond were demonstrated to retain enzymatic activity in the hydrolysis of exogenous $\mathrm{PP}$ in human synovial fluid. These results provide the basis for the further tests of PPase-based conju gates on animal models in vivo.

References:

[1] Rodina E.V., Valueva A.V., Yakovlev R.Y., Vorobyeva N.N., Kulakova I.I. Lisichkin G.V., Leonidov N.B. Immobilization of inorganic pyrophosphatase on nanodiamond particles retaining its high enzymatic activity // Biointerphases, Vol. 10, No. 4, 2015. P. 041005.

Disclosure of Interests: Anastasiya Valueva: None declared, Roman Romanov: None declared, Sofia Mariasina: None declared, Maxim Eliseev Speakers bureau: Novartis, Menarini Group, Alium, Elena Rodina: None declared DOI: 10.1136/annrheumdis-2020-eular.5321

\section{AB0936}

CLINICAL STUDY ON PERIPHERAL BLOOD IMMUNE FUNCTION IN PATIENTS WITH GOUTY ARTHRITIS

H. Wang ${ }^{1}$, H. Gao ${ }^{1} .{ }^{1}$ The Second Hospital of Shanxi Medical University, Rheumatology, Taiyuan, China

Background: Gout is an inflammatory disease characterized by hyperuricemia and recurrent arthritis. In severe cases, joint disability and renal insufficiency may occur ${ }^{[1]}$. In recent years, many studies have found that immune dysfunction plays an important role in the occurrence and development of gout ${ }^{[2]}$. Therefore, 
in-depth study of its internal mechanism is of great significance for the prevention and treatment of gout.

Objectives: This paper mainly discussed the expression of peripheral blood immune function in patients with gouty arthritis and the changes and significance of peripheral blood immune function in gout with different uric acid levels.

Methods: A retrospective analysis was performed on 258 outpatients and inpatients with gout in shanxi medical university from 2016 to 2019, all of which met the diagnostic criteria of the American college of rheumatology (ACR) in 1997, and 41 healthy controls. Complete clinical data and general laboratory data were collected, and peripheral blood lymphocyte and CD4+T cell counts were completed for all subjects.

Results: (1) Total peripheral blood B cells of gout patients [238.00 $(171.50,323.07)$ and $191.04(149.66,253.14), Z=-2.759, P=0.006]$, Th cells [814.11 $(617.50,1052.89)$ and $625.84(562.52,750.15), Z=-3.905, P<0.001], T h /$ Ts $[1.4(1.04,2.00)$ and $1.11(0.89,1.52), Z=-2.862$, Th17/Treg $[0.36(0.20,0.60)$ and $0.24(0.14,0.34), Z=-3.949, P=0.000]$ and the absolute counts of Th17 cells [9.06 $(5.07,15.57)$ and $7.48(4.31,10.18), Z=-2.520, P=0.012]$ were higher than those of the healthy control group, and the differences were statistically significant. The absolute count of Treg cells [28.82 (17.48,38.04) and $30.22(22.74,39.46)$, $\mathrm{Z}=-2.249, \mathrm{P}=0.025]$ was lower than that of the healthy control group, and the difference was statistically significant. (2) The Th17\% [1.05 $(0.71,1.42)$ and 1.27 $(0.73,2.00), Z=-1.995, P=0.046]$ and the Th17/Treg $[0.25(0.14,0.44)$ and 0.39 $(0.23,0.63), Z=-3.147, P=0.002$ ] in peripheral blood of patients with high uric acid in the gout group were higher than those in the normal uric acid group, the difference was statistically significant. The Treg \% [3.84 $(2.65,5.02)$ and 3.12 (2.36,4.37), $Z=-2.239, P=0.025]$, and the Treg cells $[30.75(21.97,43.27)$ and $24.07(16.84,36.29), Z=-2.522, P=0.012]$ were lower than those in the uric acid control group, with statistically significant differences.

Conclusion: The level of Th17 cells in peripheral blood of patients with gout increased significantly while the level of regulatory $T$ cells decreased significantly. Th17 cell level in peripheral blood of the high uric acid group was significantly increased compared with the normal uric acid group, while the regulatory $T$ cell level was also significantly decreased, and the Th17/Treg ratio was also increased. This suggests that regulatory $T$ cells may play an important role in the pathogenesis of gout and are closely related to uric acid metabolism, so the study of internal mechanism can provide a new target for the treatment of gout.

References:

[1] Ragab G, Elshahaly M, et al. Gout: An old disease in new perspective - A review[J]. Adv Res. 2017;8(5):495-511. DOI: 10.1016/j.jare.2017.04.008.

[2] Dai XJ, Tao JH, et al. Changes of Treg/Th17 Ratio in Spleen of Acute Gouty Arthritis Rat Induced by MSU Crystals. [J]. Inflammation. 2018;41(5):19551964. Doi: 10.1007/s10753-018-0839-y.

Disclosure of Interests: None declared

DOI: 10.1136/annrheumdis-2020-eular.1594

\section{Infection-related rheumatic diseases}

\section{AB0937 COMPARATIVE STUDY OF PYOGENIC SPONDYLODISCITIS VERSUS TUBERCULOUS SPONDYLODISCITIS}

A. Ben Tekaya ${ }^{1}$, L. Ben Ammar², M. Ben Hammamia ${ }^{3}$, O. Saidane ${ }^{1}$, S. Bouden ${ }^{1}$, R. Tekaya ${ }^{1}$, I. Mahmoud ${ }^{1}$, L. Abdelmoula ${ }^{1} .{ }^{1}$ Charles Nicolle Hospital,

Rheumatology, Tunis, Tunisia; ${ }^{2}$ Kassab Institute, Rheumatology, Tunis, Tunisia; ${ }^{3} \mathrm{La}$ Rabta Hospital, Cardiovascular Surgery, Tunis, Tunisia

Background: Infectious spondylodiscitis is a life-threatening infection of the intervertebral disc and adjacent vertebrae that can be caused by a variety of microorganisms. Our country is at intermediate endemicity for Tuberculosis.

Objectives: To compare the clinical, biological, radiological characteristics, management and prognosis of pyogenic versus tuberculous spondylodiscitis.

Methods: Retrospective study of 89 patients admitted to our department over a period of 20 years [1998-2018]. The diagnosis of spondylodiscitis was made based on clinical, biological, radiological and bacteriological data.

Results: The average age of the patients was 56.1 years. There were 46 men $(51.6 \%)$ and 43 women $(48.4 \%)$ with a female predominance during tuberculous spondylodiscitis $(57.1 \%)$ versus $26.9 \%$ during pyogenic spondylodiscitis ( $p$ $=0.009$ ).

Diabetes was more frequent during pyogenic spondylodiscitis but with no statistically significant difference $(p=0.4)$. The evolution time was statistically greater during tuberculous spondylodiscitis $(p<0.001)$. Patients with tuberculous spondylodiscitis had more frequently an impaired general condition $(p=0.02)$.

Hyperleukocytosis was noted more frequently in the pyogenic group than in the tuberculosis group $(p=0.03)$, while the increase in sedimentation rate was not significantly different between the two groups $(90 \mathrm{~mm} / \mathrm{h}$ and $76 \mathrm{~mm} / \mathrm{h}$, respectively, $p=0.1$ ). We found no statistically significant difference regarding the site of spondylodiscitis.

Radiologically, the frequency of para-vertebral and psoas abscesses, epiduritis and the presence of spinal cord compression were similar in the two groups $(p=0.2 ; p=0.1$ and $p=0.1$, respectively), whereas mirrored geodes were more frequent during tuberculosis $(p=0.04)$.

Surgical and interventional treatments (percutaneous sampling, abscess drainage) were more frequently noted during pyogenic spondylodiscitis, but without significant difference $(p=0.2)$. The occurrence of immediate complications was more frequent during tuberculosis but without a statistically significant difference $(\mathrm{p}=0.2)$.

Conclusion: In our series, patients with tuberculous spondylodiscitis tend to have a chronic pattern of progression and more often an impaired general condition. However, there was no significant difference in the presence of abscess, epiduritis and the occurrence of complications between tuberculous spondylodiscitis and pyogenic spondylodiscitis.

Disclosure of Interests: None declared

DOI: 10.1136/annrheumdis-2020-eular.4834

\section{AB0938 PARTICULARITIES OF INFECTIOUS SPONDYLODISCITIS INTHE ELDERLY: COMPARATIVE STUDY WITH THE YOUNG SUBJECT}

L. Ben Ammar ${ }^{1}$, A. Ben Tekaya ${ }^{2}$, M. Ben Hammamia ${ }^{3}$, O. Saidane², S. Bouden², R. Tekaya ${ }^{2}$, I. Mahmoud ${ }^{2}$, L. Abdelmoula ${ }^{2}{ }^{1}{ }^{1}$ Kassab Institute, Rheumatology, Tunis, Tunisia; ${ }^{2}$ Charles Nicolle Hospital, Rheumatology, Tunis, Tunisia; ${ }^{3}$ La Rabta Hospital, Cardiovascular Surgery, Tunis, Tunisia

Background: The incidence of infectious spondylodiscitis is steadily increasing. The prognosis depends on various parameters, including age.

Objectives: To explore the differences in presentation and the results of further investigations and the prognosis of spondylodiscitis between young and elderly subjects.

Methods: This is a retrospective study of 113 patients admitted to our department over a period of 20 years [1998-2018]. The epidemiological, clinical, biological, radiological and progressive aspects of spondylodiscitis in subjects over the age of 65 were compared to cases of spondylodiscitis in young subjects whose age is less than 65 years.

Results: Of the 113 identified patients, $33(29.2 \%)$ were classified as older, with male predominance in both groups ( 56.2 and $51.5 \%$ respectively; $p=0.6$ ). The presence of risk factors, particularly diabetes, was more frequent in the older subjects but without a statistically significant difference $(p=0.2)$. Hepathopathies were also more frequent in the elderly but without statistically significant difference $(p=0.3)$. The mean duration of progression was 3 months with no statistically significant difference between the two groups ( 4 and 5 months respectively, $p=0.1$ ). The presence of neurological symptoms, especially radiculalgia, was more frequently reported in younger subjects $(p=0.01)$. Also, young subjects were more likely to have a fever $(p=0.005)$. On the other hand, a biological inflammatory syndrome was more frequently encountered in older subjects $(p=0.03)$.

We found no statistically significant difference in the location of spondylodiscitis $(p=0.4)$. Also, multifocal involvement was more frequent in older subjects but without a statistically significant difference.

Radiologically, para-vertebral abscesses, epiduritis and spinal cord compression were more common in the elderly group $(p=0.03 ; p=0.01$ and $p=0.01$, respectively). While the frequency of intervertebral pinching and erosions was similar in both groups ( $p=0.1 ; p=0.8$, respectively).

Tuberculosis was the most involved germ in more than $50 \%$ of cases with no statistically significant difference between the two groups (57.5 and $51.5 \%$ respectively; $p=0.5$ )

All patients received targeted antibiotic therapy.

The occurrence of immediate complications was more frequent in the elderly but without a statistically significant difference $(p=0.1)$. They included neurological complications (spinal cord compression and Cauda equina syndrome) that occurred in $6.3 \%$ of cases in younger subjects versus $36.4 \%$ of cases in the elderly $(p=0.07)$. Followed by treatment side effects dominated by disturbance of liver function due mainly to antituberculosis drugs occurring in $16.3 \%$ of cases in the younger group and $15.2 \%$ of cases in older subjects $(p=0.8)$. Finally, there were 2 deaths in each group $(\mathrm{p}=0.5)$.

Conclusion: Our series has shown that the picture of spondylodiscitis in the elderly is less noisy. However, the neurological damage is more pronounced on imaging. The misleading symptomatology in the elderly explains the delay in diagnosis and treatment, which leads to more frequent complications and excess mortality.

Disclosure of Interests: None declared

DOI: 10.1136/annrheumdis-2020-eular.4855 